\title{
Leptospirose bovina no Estado da Paraíba: prevalência e fatores de risco associados à ocorrência de propriedades positivas ${ }^{1}$
}

\author{
Carla L.R.M. Pimenta² ${ }^{2}$ Vanessa Castro ${ }^{3}$, Inácio J. Clementino² ${ }^{2}$ Clebert J. Alves ${ }^{2}$, Leíse \\ G. Fernandes ${ }^{2}$, Arthur W.L. Brasil ${ }^{2}$, Carolina S.A.B. Santos ${ }^{2}$ e Sérgio S. Azevedo ${ }^{2 *}$
}

\begin{abstract}
Pimenta C.L.R.M., Castro V., Clementino I.J., Alves C.J., Fernandes L.G., Brasil A.W.L., Santos C.A.S.B. \& Azevedo S.S. 2014. [Bovine leptospirosis in Paraíba State: prevalence and risk factors associated with the occurrence of positive herds.] Leptospirose bovina no Estado da Paraíba: prevalência e fatores de risco associados à ocorrência de propriedades positivas. Pesquisa Veterinária Brasileira 34(4):332-336. Unidade Acadêmica de Medicina Veterinária, Centro de Saúde e Tecnologia Rural, Universidade Federal de Campina Grande, Av. Universitária s/n, Cx. Postal 61, Santa Cecília, Patos, PB 58700-970, Brazil. E-mail: sergio@vps.fmvz.usp.br

Herd-level and animal-level prevalences were determined and risk factors associated with leptospirosis were identified in cows in the State of Paraíba, Northeastern Brazil. A total of 2,317 cows with $\geq 24$ months of age from 450 herds were randomly sampled. For the serological diagnosis of Leptospira spp. infection, the microscopic agglutination test (MAT) was carried out using 24 serovars as antigens. A herd was considered positive when presented at least one seropositive animal. Of the 450 investigated herds, 398 (89.7\%; 95\% $\mathrm{CI}=86.6-92.2 \%$ ) presented at least one reactant animal at MAT to any serovar, and 1,349 (61.1\%; 95\% CI $=56.6-65.4 \%)$ animals were seropositive. Serovar Hardjo was the most prevalent in herds and animals, with frequencies of $58.17 \%$ and $54.69 \%$ respectively. Location of the herd in the Sertão (odds ratio $=3.20 ; p=0.003$ ), presence of wildlife (odds ratio $=2.89 ; \mathrm{p}=0.005$ ), not cooling milk (odds ratio $=3.83 ; \mathrm{p}=0.034$ ) and presence of flooded pastures (odds ratio $=2.36 ; \mathrm{p}<0.001$ ) were identified as risk factors for herd-level prevalence. It is concluded that leptospirosis is widely spread in cattle in State of Paraíba, which reinforces the need for increased prevention and control measures, such as vaccination of herds. According to the results of risk factors analysis it is suggested that the sanitary control before the introduction of animals, drainage of wetlands and improvement in management conditions are important measures to preventing the infection.
\end{abstract}

INDEX TERMS: Leptospira spp., leptospirosis, cattle, epidemiology, control, Northeastern Brazil.

RESUMO.- Foram determinadas as prevalências de propriedades positivas e de animais positivos e identificados fatores de risco associados à leptospirose em vacas no Estado da Paraíba, Nordeste do Brasil. Foram aleatoriamente selecionadas 2.317 vacas com idade $\geq 24$ meses, procedentes de 450propriedades. Para o diagnóstico sorológico

\footnotetext{
${ }^{1}$ Recebido em 14 de março de 2014.

Aceito para publicação em 4 de abril de 2014.

${ }^{2}$ Unidade Acadêmica de Medicina Veterinária (UAMV), Universidade Federal de Campina Grande (UFCG), Avenida Universitária s/n, Cx Postal 61, Santa Cecília, Patos, PB 58700-970, Brasil. *Autor para correspondência: sergio@vps.fmvz.usp.br

${ }^{3}$ Centro de Pesquisa e Desenvolvimento de Sanidade Animal, Instituto Biológico, São Paulo, SP 04014-002, Brasil.
}

da infecção por Leptospira spp. foi empregado o teste de soroaglutinação microscópica (SAM), utilizando-se 24 sorovares como antígenos. Uma propriedade foi considerada positiva quando apresentou pelo menos um animal soropositivo. Das 450 propriedades investigadas 398 (89,7\%; IC $95 \%=86,6-92,2 \%)$ apresentaram pelo menos um animal reagente na SAM para qualquer sorovar, e $1.349(61,1 \%$; IC $95 \%=56,6-65,4 \%$ ) animais foram soropositivos. 0 sorovar Hardjo foi o mais prevalente nas propriedades e nos animais, com frequências de 58,17\% e 54,69\%, respectivamente. Propriedade ser localizada no Sertão (odds ratio $=$ 3,20; $\mathrm{p}=0,003$ ), presença de animais silvestres (odds ratio $=2,89 ; \mathrm{p}=0,005$ ), não resfriar o leite (odds ratio $=3,83$; $\mathrm{p}=0,034$ ) e presença de pastos alagados (odds ratio $=2,36$; 
$\mathrm{p}<0,001$ ) foram identificados como fatores de riscos associados à prevalência de propriedades positivas. Conclui-se que a leptospirose encontra-se amplamente difundida em bovinos do Estado da Paraíba, o que reforça a necessidade de intensificação de medidas de prevenção e controle, como a vacinação dos rebanhos. De acordo com os resultados da análise de fatores de risco, sugere-se que o controle sanitário antes da introdução de animais, drenagem de áreas alagadas e melhora nas condições de manejo são importantes medidas para a prevenção da infecção.

TERMOS DE INDEXAÇÃO: Leptospira spp., leptospirose, bovinos, epidemiologia, controle, Nordeste do Brasil.

\section{INTRODUÇÃO}

A leptospirose é uma zoonose bacteriana causada por espiroquetas do gênero Leptospira (Faine et al. 1999). Com vasta distribuição geográfica, é evidenciada em todo o mundo e particularmente prevalente em países de clima tropical e subtropical, principalmente nos períodos de altos níveis pluviométricos (Acha \& Szyfres2001).

Nos animais de produção, a enfermidade está associada a abortamentos, nascimento de animais debilitados e natimortalidade. Nos bovinos as perdas econômicas causadas pela leptospirose estão ligadas às falhas reprodutivas como infertilidade e abortamento, bem como à queda da produção de carne e leite, além de custos com despesas de assistência veterinária, vacinas e testes laboratoriais (Faine et al. 1999). Esses animais são considerados hospedeiros de manutenção da sorovariedade Hardjo, pois possuem elevada suscetibilidade à infecção, apresentando a doença na forma crônica, caracterizada por problemas reprodutivos (Chiareli et al. 2012).

Os inquéritos sorológicos com determinação dos fatores de risco exercem um papel de relevância indiscutível no controle da leptospirose, pois permitem o conhecimento dos diferentes sorovares existentes em determinada região (Faine et al. 1999), bem como as condições associadas à maior ocorrência da infecção, o que possibilita a elaboração de medidas de prevenção e controle e a aplicação das mesmas de maneira correta e eficaz. No Estado da Paraíba, embora haja descrição de estudos epidemiológicos para leptospirose bovina, ainda não foi conduzido inquérito com base em amostragem planejada, o que permite o levantamento adequado de indicadores epidemiológicos da infecção.

Dessa maneira, o objetivo do presente trabalho foi determinar a prevalência de propriedades positivas e de bovinos soropositivos para Leptospira spp. no Estado da Paraíba, bem como identificar os fatores de risco associados à ocorrência de focos.

\section{MATERIAL E MÉTODOS}

Divisão do Estado da Paraíba em estratos amostrais. 0 Estado da Paraíba foi dividido em três circuitos produtores (estratos amostrais): circuito produtor 1 (Mesorregião do Sertão), circuito produtor 2 (Mesorregião da Borborema) e circuito produtor 3 (Mesorregiões da Zona da Mata e Agreste) (Fig.1). Para essa estratificação, foi considerada a capacidade operacional do Serviço de Defesa Agropecuária do Estado da Paraíba para a condução do trabalho de campo, baseando-se nas áreas de atuação das suas unidades regionais.

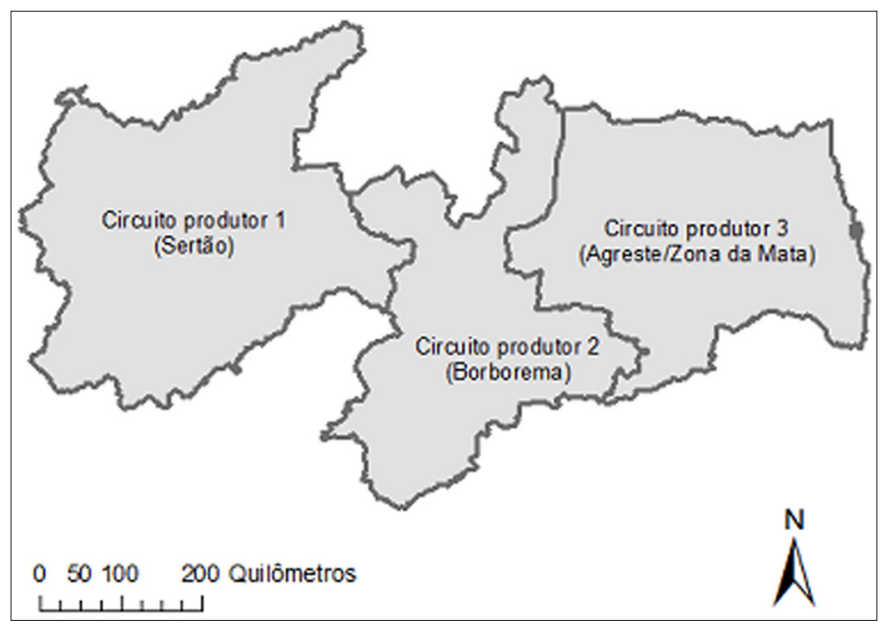

Fig.1. Mapa demonstrando a divisão do Estado da Paraíba em circuitos produtores.

População de estudo. Foi utilizado o banco de soro e o banco de dados oriundos do estudo da situação epidemiológica da brucelose bovina no Estado da Paraíba, realizado como parte do Programa Nacional de Controle e Erradicação da Brucelose e Tuberculose (PNCEBT) e planejado pelo Ministério da Agricultura, Pecuária e Abastecimento (MAPA), em colaboração com o Serviço de Defesa Agropecuária do Estado da Paraíba. A amostragem foi delineada para a determinação da prevalência de propriedades positivas e de animais soropositivos, sendo selecionadas inicialmente as unidades primárias de amostragem (propriedades), e dentro delas, as unidades secundárias (animais), conforme metodologia descrita por Silva et al. (2012).

Foram analisados 2.317 soros de fêmeas bovinas com idade igual ou superior a 24 meses, provenientes de 450 propriedades, sorteadas dentro dos três estratos amostrais do Estado. Em propriedades com até 99 fêmeas com idade $\geq 24$ meses foram amostradas 10 fêmeas; em propriedades com 100 fêmeas ou mais foram amostradas 15; e naquelas que apresentavam menos de 10 fêmeas todas foram amostradas.

Trabalho de campo. 0 trabalho de campo foi desenvolvido no período de abril a novembro de 2012 . As atividades de campo, realizadas por médicos veterinários e técnicos do Serviço de Defesa Agropecuária do Estado da Paraíba, incluíram a colheita de sangue e aplicação de questionário epidemiológico. As amostras de sangue foram colhidas de fêmeas com idade igual ou superior a 24 meses, em volumes de $10 \mathrm{~mL}$, pela punção da veia jugular com agulha descartável e tubo com vácuo (sem anticoagulante) com capacidade de $15 \mathrm{~mL}$. Após o dessoramento, o soro foi transferido para microtubo e congelado. 0 transporte das amostras para o laboratório foi feito em caixas de isopor com gelo, com o formulário epidemiológico envolvido em plástico e fixado no lado externo da tampa.

Diagnóstico sorológico. O diagnóstico sorológico da leptospirose foi realizado com a técnica de Soroaglutinação Microscópica (SAM), de acordo com Galton et al. (1965) e Cole et al. (1973), utilizando coleção de antígenos vivos que incluiu os sorovares Icterohaemorrhagiae, Canicola, Pomona, Grippotyphosa, Wolffi, Hardjo, Andamana, Australis, Autumnalis, Bataviae, Bratislava, Butembo, Castellonis, Copenhageni, Cynopteri, Hebdomadis, Javanica, Panama, Patoc, Pyrogenes, Shermani, Tarassovi, Whitcombi e Sentot. Os soros foram triados na diluição de 1:100, e aqueles que apresentaram $50 \%$ ou mais de aglutinação foram titulados pelo exame de uma série de diluições geométricas de razão dois. $\mathrm{O}$ título do soro foi a recíproca da maior diluição que apresentou 
resultado positivo.Os antígenos foram examinados ao microscópio de campo escuro, previamente aos testes, a fim de verificar a mobilidade e a presença de auto aglutinação ou de contaminantes.Uma propriedade foi considerada positiva (foco) quando apresentou pelo menos um animal soropositivo. No animal, o provável sorovar infectante foi o que apresentou o maior título, e na propriedade, o sorovar mais frequente foi aquele que apresentou maior número de reações positivas. Os animais que apresentaram dois ou mais sorovares com títulos idênticos foram considerados positivos, porém desconsiderados para o cálculo do sorovar mais frequente.

Cálculo das prevalências. Os cálculos das prevalências de focos e de animais no Estado, e das prevalências de animais dentro das regiões foram feitos de forma ponderada, conforme preconizado por Dohoo et al. (2003).

O peso de cada propriedade no cálculo da prevalência de focos no Estado foi dado por:

$$
\text { Peso }=\frac{\text { propiedades no circuito }}{\text { propiedades amostradas no circuito }}
$$

O peso de cada animal no cálculo da prevalência de animais no Estado foi dado por:

Peso $=\frac{\text { fêmeas } \geq 24 \text { meses na propiedade }}{\text { fêmeas } \geq 24 \text { meses amostradas na }} \times \begin{gathered}\frac{\text { fêmeas } \geq 24 \text { meses no circuito }}{\text { propiedade }} \\ \text { fêmeas } \geq 24 \text { meses nas propiedades } \\ \text { amostradas na região }\end{gathered}$

Na expressão acima, o primeiro termo refere-se ao peso de cada animal no cálculo da prevalência de animais dentro de região.

Comparação das prevalências e análise de fatores de risco. Para a comparação dos circuitos produtores no relativo à prevalência de focos e de animais soropositivos foi utilizado o teste de qui-quadrado. Para a análise de fatores de risco, foram formados dois grupos de propriedades - focos e não focos - que, quando comparados entre si quanto às variáveis pesquisadas no questionário epidemiológico, permitiu medir a força da associação dessas variáveis com a presença da leptospirose. As variáveis analisadas foram: circuito produtor, tipo de exploração (corte, leite e misto), tipo de criação (confinado, semiconfinado, extensivo), número de ordenhas, uso de inseminação artificial, raça predominante, número de bovinos na propriedade, presença de outras espécies domésticas, presença de animais silvestres, compra de animais, resfriamento do leite, aluguel de pastos, utilização de pasto compartilhado, presença de pastos alagados, utilização de piquete de parição e assistência veterinária.

A análise de fatores de risco foi efetuada em duas etapas: análise univariável e análise multivariável. Na análise univariável, cada variável independente foi cruzada com a variável dependente (condição sanitária da propriedade). As que apresentaram um valor de $\mathrm{p} \leq 0,2$ pelo teste de qui-quadrado foram selecionadas para a análise multivariável, utilizando-se a regressão logística múltipla (Hosmer \& Lemeshow 2000). 0 ajuste do modelo final foi verificado com o teste de Hosmer e Lemeshow, no qual um p > 0,05 indica que o modelo está ajustado. A colinearidade entre as variáveis preditoras foi verificada por meio de análise de correlação e, para aquelas que apresentaram forte colinearidade (coeficiente de correlação $>0,9$ ), uma das duas foi excluída da análise múltipla de acordo com a plausibilidade biológica (Dohoo et al. 1996). O nível de significância adotado na análise múltipla foi de $5 \%$. As análises foram feitas considerando reações sorológicas para qualquer sorovar e para o sorovar mais prevalente. Todas as análises foram realizadas com o programa SPSS 20.0 for Windows.

\section{RESULTADOS}

No Quadro 1 são apresentados os dados censitários e da amostra estudada em cada um dos circuitos produtores. No Quadro 2 são apresentados os resultados de prevalência de focos no Estado e nos circuitos. A prevalência de focos de leptospirose bovina no Estado da Paraíba foi de $89,7 \%$ (IC $95 \%=86,6-92,2 \%$ ). 0 circuito produtor 1 foi o que apresentou maior prevalência de propriedades positivas $(93,4 \%$; IC $95 \%=88,1-96,4 \%)$, seguido dos circuitos $3(89,3 \%$; IC $95 \%=83,3-93,4 \%)$ e $2(82,6 \%$; IC $95 \%=75,6-87,9 \%)$. Houve diferença significativa apenas entre os circuitos 1 e $2(p=0,007)$. Os sorovares mais frequentes por propriedade foram Hardjo, Icterohaemorrhagiae e Australis com $58,17 \%, 17,32 \%$ e $4,58 \%$, respectivamente.

Os resultados de prevalência de bovinos sororreagentes são apresentados no Quadro 3. A prevalência no Estado foi de $61,1 \%$ (IC $95 \%=56,6-65,4 \%$ ). 0 circuito produtor 3 foi o que apresentou maior prevalência $(63,1 \%$; IC $95 \%=53,4-71,8 \%)$, seguido dos circuitos $1(62,8 \%$; IC $95 \%=57,3-67,9 \%)$ e 2 $(50,6 \%$; IC $95 \%=41,6-59,6 \%)$. Houve diferença significativa entre os circuitos 1 e $2(\mathrm{p}<0,001)$ e 2 e $3(\mathrm{p}<0,0001)$. Assim como nas propriedades, os sorovares mais frequentes por animais foram Hardjo, Icterohaemorrhagiae e Australis, com $54,69 \%, 13,28 \%$ e 4,92\%, respectivamente.

Nos Quadros 4 e 5 são apresentados os resultados das análises univariáveis dos fatores de risco para qualquer sorovar e para o sorovar Hardjo, respectivamente, com as variáveis mais associadas ( $\mathrm{p} \leq 0,2)$, e no Quadro 6 observam-se os modelos finais da regressão logística com os fatores

Quadro 1. Dados censitários da população bovina do Estado da Paraíba em 2013, segundo o circuito produtor

\begin{tabular}{lccccc}
\hline Circuito produtor & \multicolumn{2}{c}{$\begin{array}{c}\text { Propriedades com } \\
\text { atividade reprodutiva }\end{array}$} & & \multicolumn{2}{c}{$\begin{array}{c}\text { Fêmeas com idade } \\
\geq 24 \text { meses }\end{array}$} \\
\cline { 2 - 3 } & Total & Amostradas & & Total & Amostradas \\
\hline 1 & 24.356 & 151 & & 288.764 & 916 \\
2 & 11.603 & 149 & & 83.428 & 688 \\
3 & 18.398 & 150 & & 192.320 & 713 \\
Total & 54.357 & 450 & & 564.512 & 2.317
\end{tabular}

Quadro 2. Prevalência de focos de leptospirose bovina no Estado da Paraíba segundo o circuito produtor, no período de abril a novembro de 2012

\begin{tabular}{lccccc}
\hline Circuito produtor & \multicolumn{2}{c}{ Propriedades } & & Prevalência (\%) & IC 95\% (\%) \\
\cline { 2 - 3 } & Testadas & Positivas & & \\
\hline 1 & 151 & 141 & & 93,4 & {$[88,1-96,4]$} \\
2 & 149 & 123 & 82,6 & {$[75,6-87,9]$} \\
3 & 150 & 134 & & 89,3 & {$[83,3-93,4]$} \\
Estado & 450 & 398 & & 89,7 & {$[86,6-92,2]$}
\end{tabular}

Quadro 3. Prevalência de bovinos sororreagentes para leptospirose no Estado da Paraíba segundo o circuito produtor, no período de abril a novembro de 2012

\begin{tabular}{lcccc}
\hline Circuito produtor & \multicolumn{2}{c}{ Animais } & Prevalência (\%) & IC 95\% (\%) \\
\cline { 2 - 3 } & Testados & Positivos & & \\
\hline 1 & 916 & 549 & 62,8 & {$[57,3-67,9]$} \\
2 & 688 & 338 & 50,6 & {$[41,6-59,6]$} \\
3 & 713 & 462 & 63,1 & {$[53,4-71,8]$} \\
Estado & 2.317 & 1.349 & 61,1 & {$[56,6-65,4]$}
\end{tabular}


Quadro 4. Resultados da análise univariável dos fatores de risco para leptospirose bovina por qualquer sorovar no Estado da Paraíba, com as variáveis mais associadas $(p \leq 0,20)$

\begin{tabular}{lcccc}
\hline \multicolumn{1}{c}{ Variável } & Categoria & $\begin{array}{c}\text { Total de } \\
\text { propriedades }\end{array}$ & $\begin{array}{c}\text { Número de } \\
\text { focos (\%) }\end{array}$ & p \\
\hline Região & Borborema & 149 & $123(82,6)$ & \\
& Agreste/Mata & 150 & $134(89,3)$ & \\
Tipo de Exploração & Sertão & 151 & $141(93,4)$ & 0,012 \\
& Corte & 50 & $47(94,0)$ & \\
& Leite & 130 & $118(90,8)$ & \\
Número de ordenhas & Mista & 270 & $233(86,3)$ & 0,181 \\
& Não ordenha & 296 & $255(86,1)$ & \\
Presença de ovinos/ & 1vez/dia & 108 & $98(90,7)$ & \\
caprinos & 2 ouezes/dia & 46 & $45(97,8)$ & 0,049 \\
Presença de animais & Sim & 277 & $239(86,3)$ & \\
silvestres & Não & 173 & $159(91,9)$ & 0,096 \\
Resfriamento do leite & Sim & 161 & $151(93,8)$ & 0,013 \\
& Não & 435 & $387(89,0)$ & \\
Assistência veterinária & Sim & 15 & $11(73,3)$ & 0,082 \\
& Não & 380 & $340(89,5)$ & \\
& Sim & 70 & $58(82,9)$ & 0,165
\end{tabular}

Quadro 5. Resultados da análise univariável dos fatores de risco para leptospirose bovina pelo sorovar Hardjo no Estado da Paraíba, com as variáveis mais associadas $(p \leq 0,20)$

\begin{tabular}{|c|c|c|c|c|}
\hline Variável & Categoria & $\begin{array}{c}\text { Total de } \\
\text { propriedades }\end{array}$ & $\begin{array}{l}\text { Número de } \\
\text { focos }(\%)\end{array}$ & $\mathrm{p}$ \\
\hline \multirow[t]{3}{*}{ Região } & Borborema & 149 & $39(26,2)$ & \\
\hline & Agreste/Mata & 150 & $66(44,0)$ & \\
\hline & Sertão & 151 & $73(48,3)$ & $<0,001$ \\
\hline \multirow[t]{3}{*}{ Tipo de exploração } & Corte & 50 & $16(32,0)$ & \\
\hline & Leite & 130 & $63(48,5)$ & \\
\hline & Mista & 270 & $99(36,7)$ & 0,040 \\
\hline \multirow{2}{*}{$\begin{array}{l}\text { Presença de ovinos/ } \\
\text { caprinos }\end{array}$} & Não & 277 & $99(35,7$ & \\
\hline & Sim & 173 & $79(45,7)$ & 0,046 \\
\hline \multirow[t]{2}{*}{ Compra de animais } & Não & 361 & $135(37,4)$ & \\
\hline & Sim & 89 & $43(48,3)$ & 0,077 \\
\hline \multirow[t]{2}{*}{ Resfriamento do leite } & Não & 435 & $176(40,5)$ & \\
\hline & Sim & 15 & $2(13,3)$ & 0,065 \\
\hline \multirow{2}{*}{$\begin{array}{l}\text { Presença de pastos } \\
\text { alagados }\end{array}$} & Não & 285 & $92(32,3)$ & \\
\hline & Sim & 163 & $86(52,1)$ & $<0,001$ \\
\hline
\end{tabular}

Quadro 6. Fatores de risco para leptospirose bovina por qualquer sorovar e pelo sorovar Hardjo no Estado da Paraíba, no período de abril a novembro de 2012

\begin{tabular}{lccc}
\hline \multicolumn{1}{c}{ Variável } & Odds ratio & IC 95\% & $\mathrm{p}$ \\
\hline Qualquer sorovar & & & \\
Propriedade ser localizada no Sertão & 3,20 & {$[1,47-6,99]$} & 0,003 \\
Presença de animais silvestres & 2,89 & {$[1,38-6,05]$} & 0,005 \\
Não resfriar o leite produzido & 3,83 & {$[1,11-13,32]$} & 0,034 \\
Sorovar Hardjo & & & \\
Propriedade ser localizada no Sertão & 2,86 & {$[1,73-4,74]$} & $<0,001$ \\
Não resfriar o leite produzido & 5,84 & {$[1,25-27,26]$} & 0,025 \\
Presença de pastos alagados & 2,36 & {$[1,56-3,56]$} & $<0,001$
\end{tabular}

de risco. Propriedade ser localizada no Sertão (odds ratio =3,20; IC 95\% =1,47-6,99), presença de animais silvestres (odds ratio $=2,89$; IC $95 \%=1,38-6,05$ ) e não resfriar o leite produzido (odds ratio = 3,83; IC 95\% =1,11-13,32) foram identificados como fatores de risco para a infecção por qualquer sorovar. Para o sorovar Hardjo, além das vari- áveis propriedade ser localizada no Sertão e não resfriar o leite produzido, a presença de pastos alagados (odds ratio =2,36; IC 95\%=1,56-3,56; $\mathrm{p}<0,001$ ) foi identificada como fator de risco. Os modelos finais apresentaram bom ajuste ( $p>0,05$; teste de Hosmer e Lemeshow).

\section{DISCUSSÃO}

As vacinas anti-leptospirose disponíveis no mercado são bacterinas inativadas baseadas na proteção dirigida ao antígeno LPS das leptospiras, ressaltando-se sua interferência na SAM por cerca de seis meses após a vacinação (De Nardi Jr et al. 2006), o que poderia resultar em reações falso-positivas no presente trabalho, uma vez que a informação de vacinação contra leptospirose não foi investigada. Porém, no Estado da Paraíba, a vacinação de bovinos contra leptospirose não é prática disseminada, fato que exclui uma possível interferência dos resultados da sorologia. Por outro lado, essa prática de não vacinar os rebanhos poderia justificar as altas prevalências (de propriedades positivas e de animais soropositivos) observada no presente trabalho. Levantamentos sorológicos recentes realizados no Brasil evidenciaram elevadas prevalências para Leptospira spp., como os de Oliveira et al. (2010), na Bahia, Castro et al. (2008), em São Paulo, e Silva et al. (2012), no Maranhão, que relataram prevalências de $77,9 \%, 71,3 \%$ e $64,81 \%$, respectivamente. Essa semelhança entre os resultados obtidos nos vários estudos de prevalência da leptospirose em bovinos indicam a necessidade da intensificação de medidas de prevenção e controle, em particular a vacinação, por ser um método eficaz e prático, contribuindo para a redução da disseminação da leptospirose, uma vez que grande parte dos problemas reprodutivos e, consequentemente, as perdas econômicas ocasionadas podem estar relacionadas à enfermidade.

O sorovar Hardjo foi considerado o mais prevalente nas propriedades e nos animais, corroborando os resultados de inquéritos sorológicos recentes realizados em bovinos no Brasil (Tonin et al. 2010, Hashimoto et al. 2012, Herrmann et al. 2012), e sua identificação como predominante nos rebanhos indica que estão presentes os mecanismos de transmissão de bovino a bovino (Figueiredo et al. 2009). De fato, este sorovar é considerado adaptado aos bovinos (Faine et al. 1999), os quais atuam como reservatórios, mantendo a infecção no rebanho.

Weschenfelder et al. (2005), Tonin et al. (2010), Hermann et al. (2012) e Silva et al. (2012) também observaram reações positivas para os sorovares Icterohaemorrhagiae e Australis, apontados como o segundo e o terceiro mais frequentes, nas propriedades e nos animais, no presente trabalho. A ocorrência destes sorovares, que são mantidos por roedores silvestres, provavelmente deve-se ao contato indireto com água contaminada com urina desses animais, uma vez que os bovinos tem acesso a aguadas (Lilenbaum, 1996). Segundo Cubas et al.(2007), o rato-de-esgoto (Rattus norvegicus) e o preá (Cavia aperea) são importantes reservatórios da sorovariedade Icterohaemorrhagiae, sendo este último um roedor amplamente encontrado no Nordeste do Brasil, e o rato d'água (Nectomys squamipes) é um dos principais reservatórios do sorovar Australis. 
A propriedade ser localizada no Sertão foi apontado como fator de risco para a leptospirose por qualquer sorovar e pelo sorovar Hardjo, e isto pode ser justificado por esta ser uma região de fronteira entre os estados do Rio Grande do Norte, Ceará e Pernambuco,onde ocorre um intenso trânsito de animais, muitas vezes sem o conhecimento da condição sanitária do rebanho de origem. A introdução de animal infectado é considerado um fator de risco (Genovez et al. 2011) e uma forma importante de introdução e dispersão da leptospirose. A presença de animais silvestres como fator de risco aponta para a importância da fauna silvestre na epidemiologia da leptospirose, uma vez que estes animais atuam como reservatórios naturais de leptospiras (Faine et al. 1999). De fato, embora o Estado da Paraíba esteja localizado na região semiárida do Nordeste, onde as condições ambientais são adversas para a sobrevivência de leptospiras, vale ressaltar que outros fatores podem influenciar na ocorrência de animais infectados, tais como as espécies animais (domésticos, selvagens ou sinantrópicos) que possam compartilhar o ecossistema, as práticas de manejo adotadas nos rebanhos, e as oportunidades de infecção direta ou indireta (Alves et al. 2000).

Não resfriar o leite foi considerado fator de risco, e isto pode estar relacionado a não tecnificação das propriedades. De fato, em propriedades tecnificadas, ou seja, que utilizam inseminação artificial, realizam ordenha mecânica e resfriam o leite produzido, os manejos produtivo e reprodutivo, incluindo medidas higiênico-sanitárias, são adequados, o que pode ter influência na diminuição da disseminação do agente no rebanho.

A presença de pastos alagados foi um fator de risco para leptospirose pelo sorovar Hardjo. A água tem um papel primordial na difusão e manutenção das leptospiras na natureza e assume uma particular importância na transmissão da doença, que ocorre por meio do contato com a água contaminada (Côrtes 1993), e estando o agente presente no ambiente, ele pode permanecer viável por longos períodos, dependendo das condições de umidade, temperatura e sombreamento, aumentando de forma significativa a chance de contato e infecção de um novo indivíduo susceptível (Faine et al. 1999).

\section{CONCLUSÃO}

Conclui-se que a leptospirose encontra-se amplamente difundida em bovinos do Estado da Paraíba, o que reforça a necessidade de intensificação de medidas de prevenção e controle, como a vacinação dos rebanhos. De acordo com os resultados da análise de fatores de risco, sugere-se que o controle sanitário antes da introdução de animais, drenagem de áreas alagadas e melhora nas condições de manejo são importantes medidas para a prevenção da infecção.

\section{REFERÊNCIAS}

Acha P.N. \& Szyfres B. 2001. Zoonosis y enfermedades transmisibles comunes al hombre y a los animales. Bacteriosis y micosis. Vol.1. 3 ${ }^{\mathrm{a}}$ ed. Organización Panamericana de La Salud, Washington, DC. 398p.

Alves C.J., Andrade J.S.L., Vasconcellos S.A., Morais Z.M., Azevedo S.S. \& Santos F.A. 2000. Avaliação dos níveis de aglutininas anti-Leptospira spp. em cães no município de Patos/PB, Brasil. Revta Bras. Ciênc. Vet. 7(2):17-21.

Castro V., Azevedo S.S., Gotti T.B., Batista C.S.A., Gentili J., Morais Z.M., Souza G.O., Vasconcellos S.A. \& Genovez M.E. 2008. Soroprevalência da leptospirose em fêmeas bovinas em idade reprodutiva no Estado de São Paulo, Brasil. Arq. Inst. Biológico, São Paulo, 75(1):3-11.

Chiareli D., Cosate M.R.V., Moreira E.C., Leite R.C., Lobato F.C.F., Silva J.A., Teixeira J.F.B. \& Marcelino A.P. 2012. Controle da leptospirose em bovinos de leite com vacina autógena em Santo Antônio do Monte, Minas Gerais. Pesq. Vet. Bras. 32(7):633-639.

Cole J.R., Sulzer C.R. \& Pulssely P.R. 1973. Improved microtechnique for the leptospiral microscopic agglutination. Appl. Microbiol. 28(5):976-980.

Cortês J.A. 1993. Epidemiologia: conceitos e princípios fundamentais. Varela, São Paulo. 227p.

Cubas Z.S., Silva J.C.R. \& Catão-Dias J.L. 2007. Tratado de Animais Selvagens: medicina veterinária. Roca, São Paulo. 1376p.

De Nardi Jr. G., Ribeiro M.G., Vasconcelos S.A., Megid J., Jorge A.M.,Geronuti L. \& Moraes Z.M. 2006. Perfil de aglutininas anti-Leptospira em bezerras búfalas vacinadas com bacterina pentavalente comercial contra leptospirose. Arq. Bras. Med. Vet. Zootec. 58(3):299-304.

Dohoo I.R., Ducrot C., Fourichon C., Donald A. \& Hurnik D. 1996. An overview of techniques for dealing with large numbers of independent variables in epidemiologic studies. Prev. Vet. Med. 29(3):221-239.

Dohoo I.R., Martin S.W. \& Stryhn H. 2003. Veterinary Epidemiologic Research. AVC Incorporated, Charlottetown. 706p.

Faine S., Adler B., Bolin C. \& Perolat P. 1999. Leptospira and leptospirosis. $2^{\text {nd }}$ ed. MediSci, Melbourne. 272p.

Figueiredo A.O., Pellegrin A.O., Gonçalves V.S.P., Freitas E.B., Monteiro L.A.R.C., Oliveira J.M. \& Osório A.L.A.R. 2009. Prevalência e fatores de risco para a leptospirose em bovinos de Mato Grosso do Sul. Pesq. Vet. Bras. 29(5):375-381.

Galton M.M., Sulzer C.R., Santa Rosa C.A. \& Fields M.J. 1965. Application of a microtechnique to the agglutination test for leptospiral antibodies. Appl. Microbiol. 13(1):81-85.

Genovez M.E., Escócio C., Castro V., Gabriel F.H.L., Chiebao D.P. \& Azevedo S.S. 2011. Fatores de risco associados à infecção pela Leptospira spp. sorovar Hardjo em rebanhos exclusivos de ovinos e nos consorciados com bovinos. Arq. Inst. Biológico, São Paulo, 78(4):587-592.

Hashimoto V.Y., Dias J.A., Spohr K.A.H., Silva M.C.P., Andrade M.G.B., Muller E.E. \& Freitas J.C. 2012. Prevalência e fatores de risco associados à Leptospira spp. em rebanhos bovinos da região centro-sul do estado do Paraná. Pesq. Vet. Bras. 32(2):99-105.

Herrmann G.P., Rodrigues R.O., Machado G., Lage A.P., Moreira E.C. \& Leite R.C. 2012. Soroprevalência de leptospirose em bovinos nas Mesorregiões Sudeste e Sudoeste do Estado Rio Grande do Sul, Brasil. Ciênc. Anim. Bras. 13(1):131-138.

Hosmer D.W. \& Lemeshow S. 2000. Applied logistic regression. John Wiley and Sons, New York. 375p.

Lilenbaum W. 1996. Atualização em leptospiroses bovinas. Rev. Bras. Med. Vet. 18(1):9-13.

Oliveira F.C.S., Azevedo S.S., Pinheiro S.R., Batista C.S.A., Moraes Z.M., Souza G.O., Gonçalves A.P. \& Vasconcellos S.A. 2010. Fatores de risco para a leptospirose em fêmeas bovinas em idade reprodutiva no Estado da Bahia, Nordeste do Brasil. Pesq. Vet. Bras. 30(5):398-402.

Silva F.S., Conceição W.L.F., Fagliari J.J., Girio R.J.S., Dias R.A., Borba M.R. \& Mathias L.A. 2012. Prevalência e fatores de risco de leptospirose bovina no Estado do Maranhão. Pesq. Vet. Bras. 32(4):303-312.

Tonin A.A., Azevedo M.I., Escobar T.P., Casassola I., Santos L.G., Silva A.S., Martins J.L.R., Schaefer P.C. \& Badke M.R.T. 2010. Leptospirose bovina: aumento na incidência da Leptospira interogans sorovar Butembo no rebanho do Estado de Santa Catarina, Brasil. Acta Vet. Bras. 4(4):294-297.

Weschenfelder S., Pires Neto J.A. \& Schmidt V. 2005. Levantamento sorológico e distribuição geográfica da leptospirose em bovinos no Rio Grande do Sul, no período de 1999 a 2001. Pesq. Agropec. Gaúcha 11(1/2):89-93 